\title{
EDITORIAL
}

\section{Lung growth and computed tomography}

\author{
A.A. Hislop
}

Studies on normal lung development have shown that all airways are present by half-way through gestation, and approximately one-third to one-half of the adult number of alveoli develop during the last trimester of pregnancy [1, 2]. Modern treatment of premature babies born with immature lungs has led to the survival of a large cohort of infants and children who may have an abnormal lung or one that will not have the potential to reach the normal adult state. In addition, pre- and postnatal treatment of babies with congenitally hypoplastic lungs is now widespread. It is vital to know how these lungs will grow to establish or predict any problems of middle and old age. Very few pathological studies have been performed on either normal lung during development or abnormal cases, and for the latter only the worst affected cases are available. Lung function testing although noninvasive is still relatively time-consuming for young children and does not produce accurate information on the separate structures within the lungs.

The paper by DE JONG et al. [3] in this issue of the journal is therefore timely. In this paper for the first time, computed tomography (CT) has been used to assess lung growth. The authors have studied 35 normal children aged from 15 days to 17.6 yrs and shown close correlation between estimated lung volume and reported data of functional residual capacity, and between estimated lung density (weight) and post mortem lung weight. Previous studies on normal postnatal lung growth used quantitative analysis on lung specimens [4-6] and were based on few cases. These studies described a symmetrical increase of airway size, by measuring bronchograms [4] and multiplication of alveoli for up to 2-4 yrs, followed by an increase in size $[5,6]$. The authors suggest that by using CT scans they can estimate relative expansion of the lung. This would be particularly important in estimating the lung growth after very early premature delivery and in cases with bronchopulmonary dysplasia (BPD) where the alveoli are known to be increased in size and reduced in number compared with normal, but often within a normal total lung volume [7, 8]. This structural abnormality leads to a reduced gas exchange capacity due to decreased surface area to volume.

Most interpretation of CT scanning in children is based on extrapolation from adult studies. The approach used by DE JONG et al. [3] provides a basis to build a database of normal lung growth that will act as a baseline for assessing abnormal growth. The timing of this study is even more pertinent because of the advent of multislice/volumetric scanning with faster acquisition times and therefore less need for sedation or general anaesthesia, which are more frequently needed in the paediatric population because of respiratory and motion artefacts. Acquisition times are so rapid that they can be acquired during one respiratory excursion and hence allow precise, accurate assessment of the entire lung volume,

Correspondence: A.A. Hislop, Unit of Vascular Biology and Pharmacology, Institute of Child Health, University College London, 30 Guilford Street, London WC1N 1EH, UK. Fax: 442079052321. E-mail: A.Hislop@ich.ucl.ac.uk with superb spatial resolution when using thin slices. In future this facility will be more widely available.

Previous studies on lung growth have depended upon pathological specimens or radiographs on a small number of individuals. The lack of recent data is apparent from the era of the papers that the authors have had to use for their reference points. Such data will become even more difficult to collect in that pathological specimens are less available, and studies on them, even with semiautomated quantitative techniques, are time-consuming and tedious. Although the present CT study is cross-sectional, longitudinal studies should be possible and would allow an analysis of normal growth and the progression of a disease or its resolution. Although CT scanning is less time-consuming than lung function testing, for a longitudinal study the issue of radiation dose would have to be considered.

This study provides promising data in a new area. However, the numbers of subjects at each age group are relatively low and the cases were not separated by sex, which has an effect on lung growth. Having established that lung weight and volume can be estimated, a larger cohort studied longitudinally would produce a normal growth curve for comparison with abnormal cases. Validating the technique would require careful animal studies, in which data from CT scans could be directly related to the pathological specimen and, possibly, to information from other techniques including magnetic resonance and lung function testing.

Is this technique better or worse than magnetic resonance imaging (MRI)? On the plus side it is less expensive, more widely available and needs less specialised staff and research units as the acquisition times are very rapid, although sedation and general anaesthesia may be needed in younger children. The inherent spatial resolution of CT is superior to MRI and therefore CT is more appropriate when looking at small anatomical structures i.e. small airways and alveoli. To date, MRI of the lungs cannot replace the excellent spatial resolution of thin-section $\mathrm{CT}$, but magnetic resonance has no radiation burden and better contrast resolution than $\mathrm{CT}$.

What could CT be used for? Lung growth could be monitored in premature infants, particularly those whose lung function is thought to be normal and who have not developed BPD. Recent lung function studies suggest they are not always normal [9]. Monitoring infants with BPD or congenitally hypoplastic lungs, e.g. corrected congenital diaphragmatic hernia, would also be of interest. Monitoring lung and airway growth postlung transplant could also be useful, as would seeking evidence of obliterative bronchiolitis. In future it will be possible to assess perfusion as well as total lung volumes and large-to-small airway volume.

The paper by DE JONG et al. [3] provides optimism that the growth of the lung in a physiological setting in both infants and children can be accurately monitored in the near future. The importance of assessing long-term damage in lungs affected in infancy and the prediction for adult disease cannot be overemphasised. 
Acknowledgements. Funded by The British Heart Foundation. The author would like to thank C. Owens (Great Ormond Street Hospital) for discussion on computed tomography scanning.

\section{References}

1. Bucher U, Reid L. Development of the intrasegmental bronchial tree: the pattern of branching and development of cartilage at various stages of intra-uterine life. Thorax 1961; 16: 207-218.

2. Hislop A, Wigglesworth JS, Desai R. Alveolar development in the human fetus and infant. Early Hum Dev 1986; 13: 111.

3. de Jong PA, Nakano Y, Lequin MH, et al. Estimation of lung growth using computed tomography. Eur Respir J 2003; 22: $235-238$
4. Hislop A, Muir DCF, Jacobsen M, Simon G, Reid L. Postnatal growth and function of the pre-acinar airways. Thorax 1972; 27: 265-274.

5. Thurlbeck WM. Postnatal human lung growth. Thorax 1982; 37: 564-571.

6. Zeltner TB, Caduff JH, Gehr P, Pfenninger J, Burri PH. The postnatal development and growth of the human lung. I Morphometry. Respir Physiol 1986; 67: 247-267.

7. Hislop A, Wigglesworth JS, Desai R, Aber V. The effects of preterm delivery and mechanical ventilation on human lung growth. Early Hum Dev 1987; 15: 147-164.

8. Coalson JJ. Pathology of chronic lung disease of early infancy. In: Bland RD, Coalson JJ, eds. Chronic Lung Disease in Early Infancy. New York, Marcel Dekker, 2000; pp. $85-124$

9. Hoo AF, Dezateux C, Henschen M, Costeloe K, Stocks J. Development of airway function in infancy after preterm delivery. $J$ Pediatr 2002; 141: 652-658. 